\title{
AN ANGIOSARCOMA IN THE RIGHT ATRIUM: A CASE REPORT
}

\author{
Nicoleta F. Dumitru, \\ Daniela I. Bartos ${ }^{1}$ \\ Internal Medicine Clinic, \\ Clinical Emergency Hospital of \\ Bucharest \\ ${ }^{1}$ University of Medicine and Pharmacy \\ Carol Davila, \\ Internal Medicine Clinic, \\ Clinical Emergency Hospital of \\ Bucharest, \\ Romania
}

\begin{abstract}
Summary
Angiosarcoma is a rare malignant tumor of vascular endothelial cell origin, accounting for 1-2 \% of all sarcomas. We present a 24 -year-old female patient, initially diagnosed and treated for tuberculous pleurisy for one month. Then transthoracic echocardiography (TTE) revealed a rare cause for the pleural and pericardial effusion - a tumoral mass apparently arising from the right atrium and extending into the upper vena cava. The patient presented with worsening dyspnea, stabbing pain in the right hemithorax and persistent, irritating cough, a recent history of haemoptysis, bilateral pleurisy and hemorrhagic pericardial effusion. The ECG showed sinus rhythm with negative T-waves in leads DI, DII, aVL, V3 to V6. The TTE showed an irregularly shaped right atrial tumoral mass, not resembling a thrombus, which extended to the upper vena cava. The transesophageal echocardiography showed an invasion of the serous (parietal and visceral) pericardium and a dilated right atrium almost completely occupied by the tumoral mass. The CT scan revealed invasion of the upper vena cava ostium and anterior mediastinum with pretracheal adenopathies, and hemorrhagic pleural and pericardial effusion suggestive of a sarcoma. The histological examination and immunohistochemistry confirmed the diagnosis of angiosarcoma. Although a rare form of malignant tumor that affects the head, neck, breast, bone, liver, spleen and heart, angiosarcoma must be considered as a possible cause of pleural and pericardial effusion, especially in previously healthy young patients.
\end{abstract}

Key words: angiosarcoma, pleurisy, pericarditis

\section{Introduction}

Angiosarcomas are a heterogenous group of malignant vascular tumors with different anatomic locations, and different clinical settings (radiations, associated chronic lymphedema). They are rare tumors, representing only $1-2 \%$ of all soft tissue sarcomas that usually have an aggressive clinical course and limited management options. Soft tissue sarcomas represent an important cause of death in the age group 14-29 years [1]. Approximately half of all patients diagnosed with intermediate or high- 
grade soft tissue sarcomas develop metastatic disease requiring systemic treatment [2], the overall survival being of approximately $50 \%$ at 5 years.

\section{Case presentation}

We present the case of a 24-year-old non-smoker woman presenting to the local emergency hospital for worsening dyspnea, stabbing pain in the right hemithorax, and persistent irritating cough. The patient was initially diagnosed with right inferior lobe pneumonia and pleurisy and was treated with antibiotics and NSAIDs. The patient did not improve after this treatment, and tuberculous pleurisy was then suspected. A pleural biopsy and thoracocentesis liquid sample were collected and antituberculous treatment plus AIDs was started. Despite the treatment, the patient's status deteriorated fast: the dyspnea worsened, the irritating cough caused vomiting that rendered treatment administration impossible and made the patient extremely agitated. A pulmonary X-ray was taken, which revealed bilateral pleurisy and an enlarged cardiac shadow as a result of the pericardial effusion. The ECG (Figure 1A) performed showed signs of pericarditis - diffuse, lowvoltage and flattened or slightly inverted $\mathrm{T}$ waves, except aVR and V1. The patient was diagnosed with cardiac tamponade, presumed as tuberculous. A pericardiocentesis was performed and the effusion was evacuated. The antituberculous treatment was continued but the patient's status worsened.

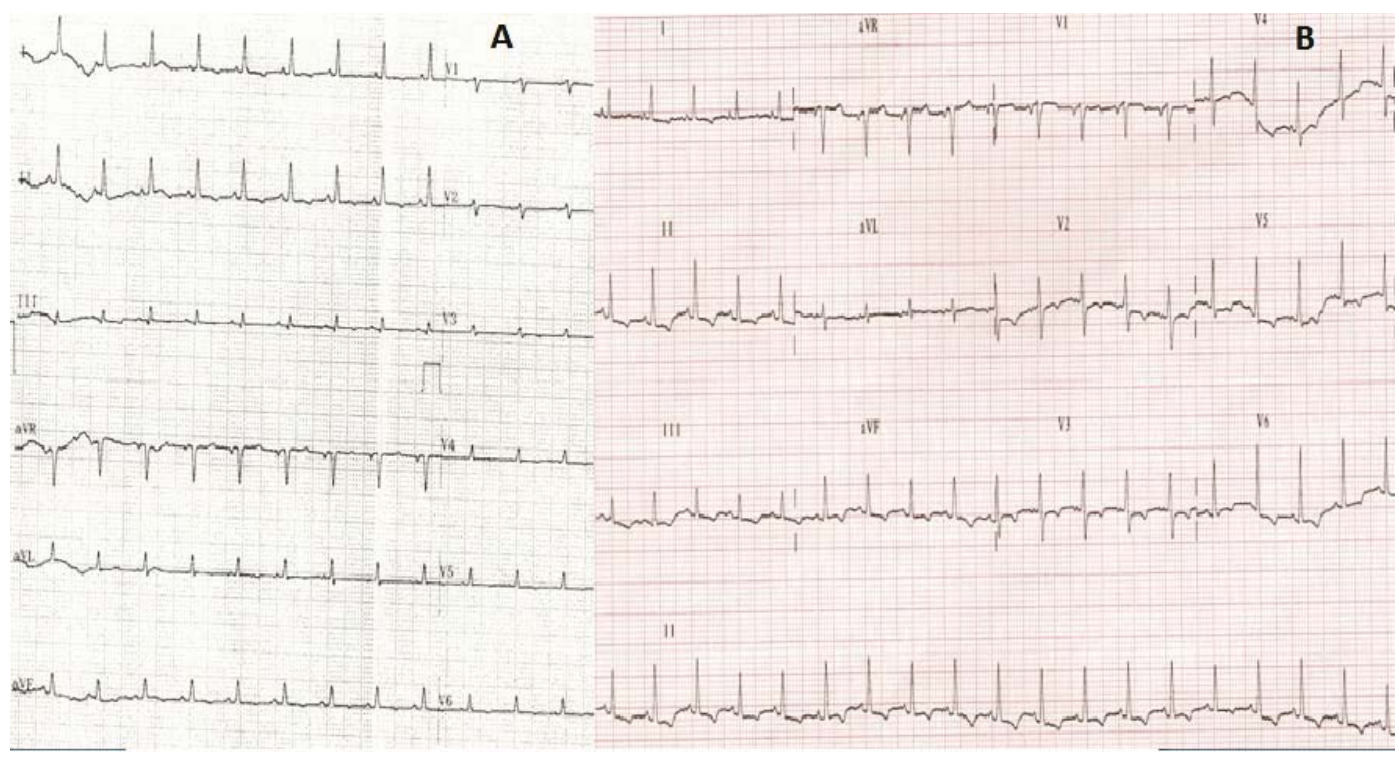

Figure 1. A - ECG, showing pericarditis with low voltage and T-flattened waves; B - ECG on arrival to the clinic showing pericarditis with electrical alternans and diffuse T negatives except a VR and V1

The patient was transferred to a Cardiology Clinic of the Emergency County Hospital. Here, pericardiocentesis revealed hemorrhagic pericardial fluid. A sample was referred for analysis and a pericardial biopsy sample was collected. The laboratory tests revealed mild anemia ( $\mathrm{Hb} 10.3 \mathrm{~g} / \mathrm{dl})$, severe inflammatory syndrome (elevated C-reactive protein, fibrinogen and erythrocyte sedimentation rate). The cultures for Mycobacterium tuberculosis (pericardial effusion liquid, sputum) were negative. The echocardiography performed to evaluate the pericardial effusion quantity revealed an immobile tumoral mass in the right atrium with a broad implantation base not suggestive of a thrombus. Because of this finding, the patient was transferred to our clinic for further evaluation of the etiology of the tumoral mass and treatment.

On arrival to the clinic, the patient presented resting dyspnea, paleness of the skin and fatigue. On clinical examination, the blood pressure was $120 / 80 \mathrm{mmHg}$ with a heart rate of 110 b.p.m, regular and no superimposed heart sounds or 
murmurs. The vesicular murmur was absent in the inferior half of the right hemithorax and inferior third of the left hemithorax suggested a bilateral pleural effusion. The liver was slightly enlarged, with no tumoral masses. The ECG (Figure 1B) presented electrical alternans and diffuse inverted T-waves except aVR (positive T wave) and V1 (biphasic), suggesting a pericardial effusion. The transesophageal echocardiography showed a dilated right atrium, occupied in a proportion of $60-70 \%$ by an echodense, polylobular tumoral mass that was adherent to the superior and posterior right atrial walls (Figure
2A) and invaded the visceral pericardium of the right atrium and possibly the parietal pericardium. The mass protruded into the superior vena cava, narrowing its lumen. It did not invade the tricuspid valve but determined the turbulent flow inside the right atria (Figure 2B). It did not invade the interatrial or interventricular septum, the left cardiac cavities having normal morphology and dimensions. There was a $9 \mathrm{~mm}$ circumferential pericardial effusion and small hyperechogenic fibrin deposits on the pericardial lamina.

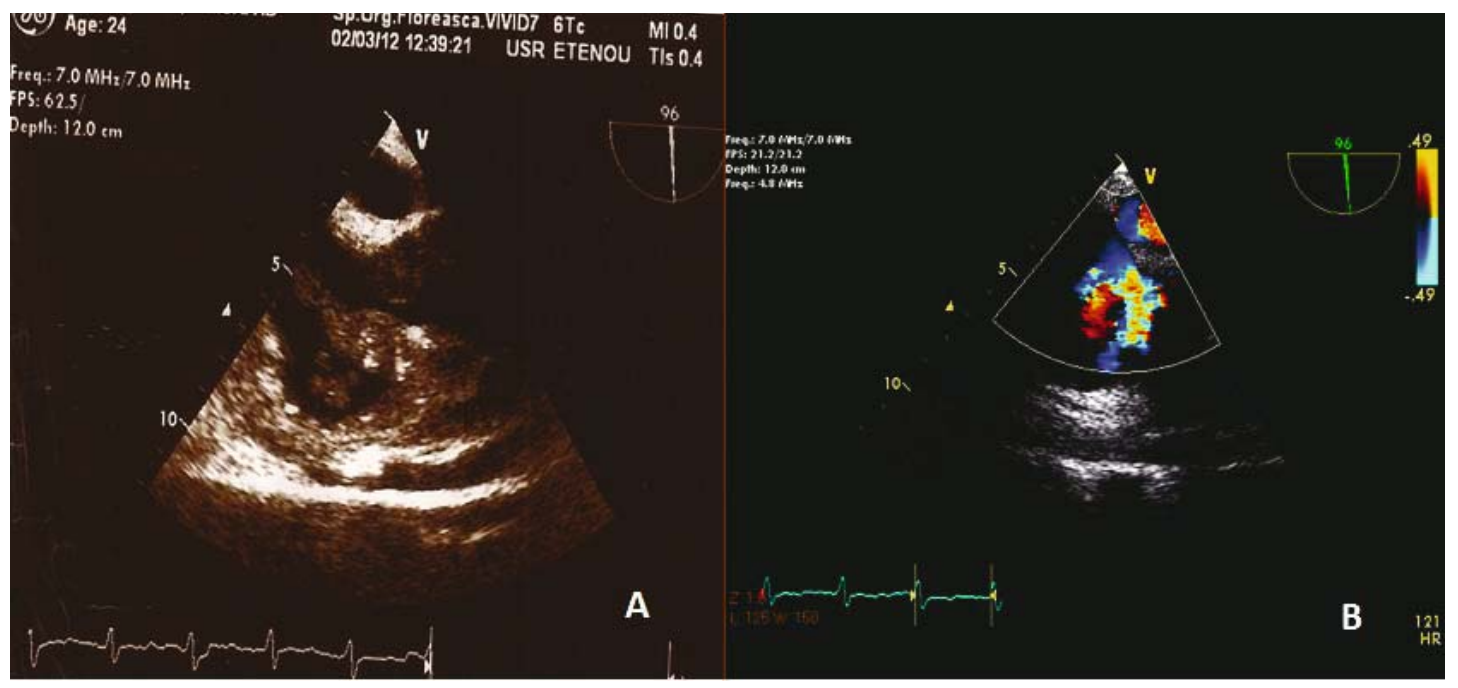

Figure 2. A - Tumoral mass of the right atrium in transesophageal echocardiography; B - Intraatrial turbulence determined by the tumoral mass

A CT scan of the head, thorax, abdomen and pelvis, both native and with intraveous contrast, was performed. It confirmed the presence of the right atrial hypodense, non-iodophilic tumoral mass of $9.4 / 7 / 4.3 \mathrm{~cm}$ that invaded the right atrial walls, the vena cava superior, the pericardium, and the mediastinal fatty tissue. There were no pathological pulmonary, cerebral, abdominal or pelvic masses. Pretracheal adenopathies were visualized. A bilateral pleural effusion of 5-6 cm and a circumferential pericardial effusion of approximately $1-1.5 \mathrm{~cm}$ were present, both of them of hemorrhagic origin (Figure 3A, Figure B4). Pericardial sarcoma with right atrial invasion or right atrial tumor (sarcoma?) with pericardial and vascular invasion was suspected.

The previously collected biopsy samples did not reveal any abnormal mitotic activity or cells but suggested a chronic inflammatory process with lymphocytosis, monocytosis and hyperemia.

The liquid samples helped establish that both effusions were exudates (total proteins $4.9 \mathrm{~g} / \mathrm{dl}$, LDH $618 \mathrm{UI} / 1$, glucose $95 \mathrm{mg} / \mathrm{dl}$ ) of noninfectious origin.

Corroborating all the data to that moment, we concluded that sarcoma was very likely but we did not know its precise origin (pericardial, angiosarcoma). Evaluation did not reveal distant metastases on evaluation but pretracheal adenopathies were present, and all of these in a young, previously healthy patient who was clinically stable at the moment. A team including an oncologist, a cardiovascular surgeon, a cardiologist, an anesthesiologist was built. After consulting the data in the literature and obtaining the patients informed consent, it was decided to perform cardiac surgery with tumor resection en 


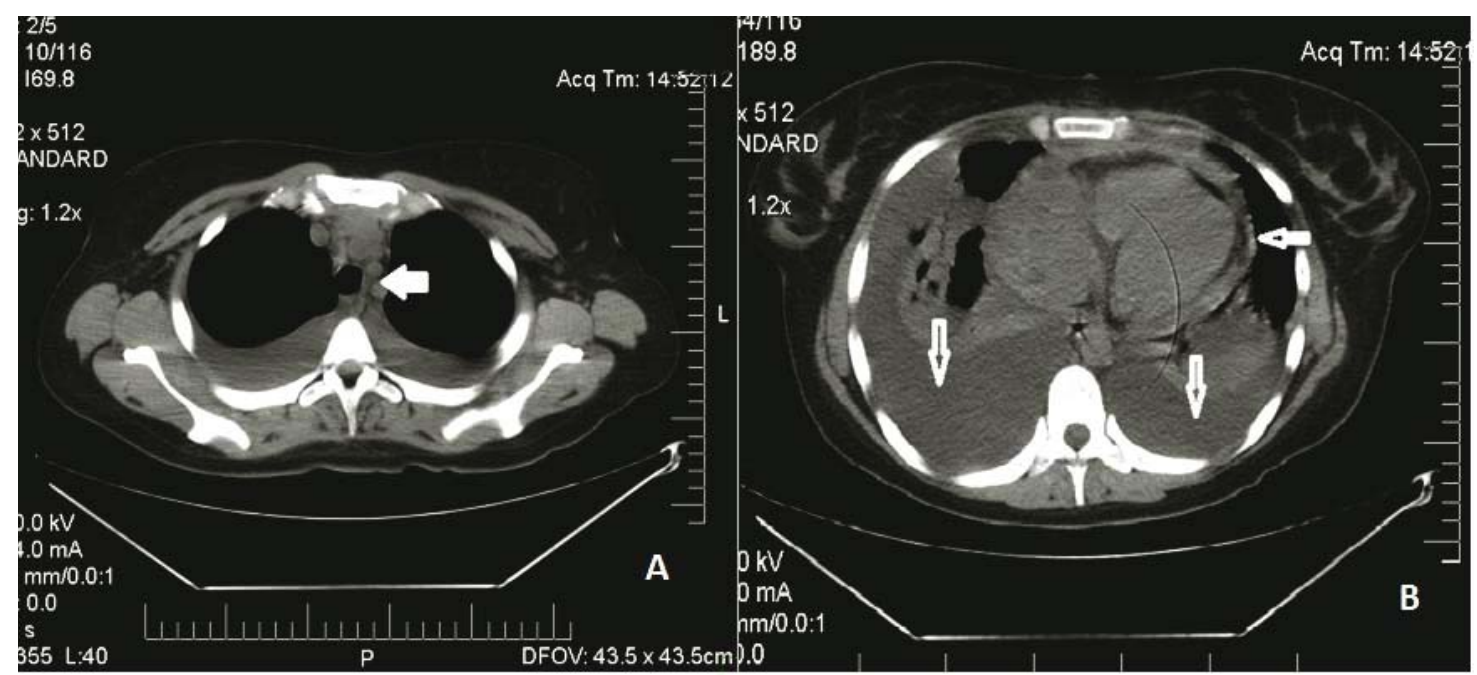

Figure 3. A - Pretracheal adenopathies in native CT scan image; B - Hemorrhagic bilateral pleural effusion and pericardial effusion

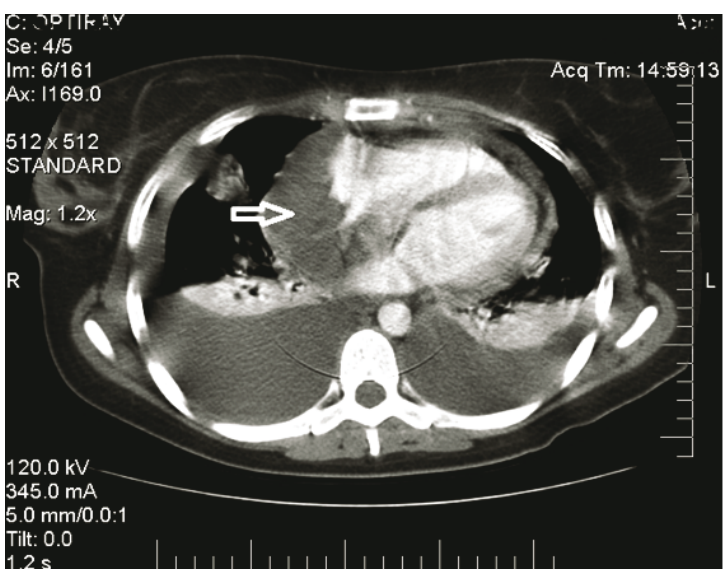

Figure 4. Tumoral mass invading the right side of the heart

bloc, followed by radiotherapy.

The surgical intervention proved to be difficult due to the extensive tumoral invasion of the right side of the heart - right atrium, superior vena cava, inferior vena cava, left atrial base, pulmonary veins. Only a partial excision of the tumoral mass was achieved, leaving macroscopic residual tumoral tissue.

Macroscopic tumoral disease is associated with a poor overall prognosis and the unlikely probability of achieving local control even with postoperative radiotherapy [2]. Histological examination of the biopsy material obtained was made. In the hemalaun-eosin (HE) staining, the histological finding was suggestive of angiosarcoma tissue fragments, consisting of vascular structures with different dimensions, with an endothelium formed by cells with round nuclei and hobnail cells with a moderate/focal pleomorphism. The immunohistochemistry analysis showed an intense positive vimentine diffuse in the tumoral cells and in the vascular walls, a negative CK 7 in tumoral cells and focal positive in the mesothelial cells (normal aspect) and a $70 \%$ or more positive Ki 67 nuclear protein (marker of proliferation). An additional cluster of differentiation markers CD 31 and CD 34 were determined as positive (vascular tumor specific). We now had a diagnosis: the tumor was an angiosarcoma. Unfortunately, the clinical status of the patient worsened rapidly after the intervention because of the large quantity of pleural and pericardial effusions that accumulated fast, with exitus in the 6th postoperative week, despite diuretic and positive inotropic intensive treatment.

The grading of the tumor was made using the Federation National des Centre de Lutte Contre le Cancer (FNCLCC) grading system. This grading system considers tumor differentiation, necrosis and the mitotic count $(\mathrm{n} / 10$ high power fields). The tumor grade using this system was 3 (high grade), associated with poor prognosis. The staging of the tumor was made using the AJCC TNM Classification for soft tissue sarcomas was made on the basis of data obtained from the CT scan. The patient had no metastases, the regional lymph nodes were invaded and the tumor was larger then $5 \mathrm{~cm}$, so the tumor was staged as T2N1M0G3. 


\section{Discussion}

The anthology of soft tissue sarcomas is unknown, and some gene mutations are incriminated in some cases. In angiosarcomas, a KDR activating mutation is studied [3].

Unfavorable prognostic factors associated with angiosarcoma are: old age, retroperitoneal location and larger tumor size. According to the soft tissue sarcoma management guidelines, the best results in the treatment of angiosarcomas have been obtained by surgery with wide margin tumor resection, followed by radiotherapy. Other treatment options are represented by neoadjuvant chemotherapy (Doxorubicin, Ifosfamide, Gemcitabine) of high-grade lesions, preoperative or postoperative radiotherapy in conjunction with surgery, with $80 \%$ local control. The problems associated are the moderate chemosensitivity of the angiosarcomas and the

\section{References}

1. Ferrari A, Bleyer A. Participation of adolescents with cancer in clinical trials. Cancer treat Rev. 2007;33(7):603-8.

2. Grimer R, Judson I, Peake D, Seddon B. Guidelines for the Management of Soft Tissue Sarcomas. Sarcoma. Volume 2010 (2010). Article ID 506182. doi:10.1155/2010/506182 wide spread of the disease at diagnosis $(50 \%$ of patients have metastases when diagnosed). In addition, radiotherapy and chemotherapy have not been proven to improve survival.

\section{Conclusions}

Therefore, the best therapeutic approach is represented firstly by angiosarcoma awareness, which is essential for making the diagnosis at an early stage. The best treatment strategy should be evaluated for each case considering the stage of the tumor, clinical presentation and the patient's status by a team of experts in the field of soft tissue sarcomas. There are few studies because of the small number of patients but new studies are about to begin evaluating the efficacy of novel therapeutic agents and novel combinations of therapeutic agents (e.g. trebananib in patients with unresectable angiosarcoma).

3. Antonescu C R, Yoshida A, Guo T, Chang NE, Zhang L, Agaram NP, et al. KDR Activating Mutations in Human Angiosarcomas Are Sensitive to Specific Kinase Inhibitors. Cancer Res. 2009,69(18);7175-9. 